\title{
Drug Utilization Review of Piperacillin/Tazobactam at a Tertiary Care Hospital, Pakistan
}

\author{
Ehsan Elahi ${ }^{1 *}$, Umar Zia ${ }^{1}$, Ayesha Rahman ${ }^{1}$, Iqra Masood ${ }^{1}$, Ayesha Tariq ${ }^{2}$, Sidrah Andleeb $^{1}$, Muhammad Tahir \\ Aziz $^{1}$ and Omar Akhlaq Bhutta ${ }^{1}$
}

${ }^{1}$ Department of Pharmaceutical Services, Shaukat Khanum Memorial Cancer Hospital, Pakistan

${ }^{2}$ Ghurki Trust Teaching Hospital, Pakistan

Submission: September 14, 2017; Published: October 26, 2017

*Corresponding author: Ehsan Elahi, Department of Pharmaceutical Services, Shaukat Khanum Memorial Cancer Hospital and Research Centre, Pakistan, Tel: 0092-322-6277796, Email: ehsan_ilahi21@hotmail.com

Abstract

Purpose: This study was conducted to estimate the rationality of Piperacillin/Tazobactam utilization in our hospital.

Methodology: This cross sectional, retrospective study was aimed to involve all those patients who were admitted to a tertiary care hospital Pakistan, and were prescribed Piperacillin/Tazobactam as an empiric therapy over a period of three months. The medical records for those patients were retrospectively reviewed and analyzed.

Results: The data came out with 76 prescriptions which were ordered for 73 patients. Main indications for utilization of Piperacillin/ Tazobactam were febrile neutropenia (34/76; 44.73\%). Overall percentage of rational and irrational use of empiric therapy was (49/76; $64.47 \%)$ and $(27 / 76 ; 35.52 \%)$ respectively (P- value 0.001). Cases of wound/surgery/skin/soft tissue infections (WSSSTI), abdominal infections and pneumonia and unit of palliative care, showed higher trends of irrational prescribing. Microbiology data was positive for $(40 / 76 ; 52.63 \%)$ prescriptions. There were $(12 / 24 ; 50 \%)$ prescriptions where antibiotic was switched after culture sensitivity reports to a narrow spectrum agent. In $(12 / 24 ; 50 \%)$ cases, de-escalation was not considered to a narrow spectrum antibiotic and Piperacillin/ Tazobactam was continued. Regarding the rationality as per criteria for dosing, dose adjustments and indications; $(26 / 39 ; 66.66 \%)$ orders fulfilled the criteria for rational use whereas $(13 / 39 ; 33.33 \%)$ prescriptions were irrational $(\mathrm{P}=0.000)$

Conclusion: This study showed that there was an unwise use of Piperacillin/Tazobactam at our hospital, as supported by significant proportion of irrationality in the view of empiric prescriptions and drug modifications even in the presence of microbial culture results.

Keywords: Drug utilization review; Piperacillin/Tazobactam; Rational use of Piperacillin/Tazobactam; Antibiotic Stewardship Program

Abbreviations: IDSA: Infectious Disease Society of America; ICU: Intensive Care Unit; HIS: Hospital Information System; FN: Febrile Neutropenia; WSSSTI: Wound/Surgery/Skin/Soft Tissue Infections; UTI: Urinary Tract Infection; CME: Continued Medical Education; ASP: Antibiotic Stewardship Program; GNB: Gram-Negative Bacilli

\section{Introduction}

Development of bacterial resistance has become a serious problem throughout the world. Resistance may be the result of many contributing factors most common being the, the irrational widespread use of antibiotics [1]. The development of broadspectrum antibiotics such as, fourth generation cephalosporin's, carbapenems and piperacillin/tazobactam (Piperacillin/ Tazobactam), has led to their use as an empirical therapy for many serious infections now-a-days. This widespread use of antibiotics or more precisely misuse of these agents; is high and costly [2].
Because mostly the health care provider goes for a broad spectrum-antibiotic even when a narrow spectrum-antibiotic would be enough [3]. This excessive dependence on these agents is thought to be a major underlying cause of bacterial resistance [4]. In order to overcome this growing problem, many hospitals execute a set of measures like designing and following antibiotic formulary and guidelines [5]. In our hospital, IDSA practice guidelines regarding antimicrobial agent use are followed $[6,7]$.

Some other methods which assist in strengthening the instructions of guidelines in our hospital include observation 
of antibiotic utilization by the infectious disease consultants/ fellows and infectious disease pharmacist, continuing education of health care providers with respect to better utilization of antimicrobial agents, application of automatic stop of medication order, and drug utilization reviews [8]. Increasing bacterial resistance and advancements in the field of infectious diseases have opened new horizons in the development of newer agents with effective as well as enhanced antibacterial spectrum.

From the time of revolutionary discovery of penicillin antibiotics which were effective against many bacterial infections caused by staphylococci and streptococci, the world is in the age of Piperacillin/Tazobactam, a $\beta$-lactam/ $\beta$-lactamase inhibitor combination with a broad spectrum of antibacterial activity against Gram (+), Gram (-) aerobic and anaerobic bacteria. Piperacillin/Tazobactam is very well tolerated and effective against patients with lower respiratory tract, intra-abdominal, skin and soft tissue infections and febrile neutropenia [9].

Many years ago, Piperacillin/Tazobactam was approved by the pharmacy and therapeutic committee of our hospital and after a short period of time it became the most prescribed antibiotic combination in our hospital. In 2013, there were a total of 2080 Piperacillin/Tazobactam prescriptions. Different hospitals assess the utilization of antibiotics by estimating quantitative pharmacy data which enables to calculate the number of defined daily doses per occupied bed-days, but it doesn't provide an indication of appropriateness whether therapy was rational or irrational. So in the view of increasing number of prescriptions and mounting expenditure, this study was conducted to estimate the rationality of Piperacillin/Tazobactam utilization in our hospital.

\section{Materials and Methods}

\section{Design and Setting}

A cross sectional, retrospective study was conducted at cancer specialty hospital. The in-patient setting comprises of surgical unit, pediatric oncology unit, adult oncology unit, ICU and palliative care unit.

\section{Data Source}

The purposive sampling technique was used to select those patients who were admitted to our hospital during a period of three months and were prescribed Piperacillin/ Tazobactam as an empiric therapy. Data in the form of medical record numbers of these patients was extracted by utilizing HIS, and then medical records of randomly selected patients were retrospectively reviewed and studied. 81 prescriptions of Piperacillin/Tazobactam were reviewed for 78 patients. Only 5 patients were prescribed Piperacillin/Tazobactam as definitive therapy of documented infections and so were excluded from the study, whereas 76 prescriptions were prescribed initially as empiric therapy of suspected infection. These 76 prescriptions were thus considered subject for this study.

\section{Criteria for appropriateness of Piperacillin/Tazobactam prescription}

In order to analyze the rationality of Piperacillin/Tazobactam prescriptions, a set of criteria was established by which a prescription was regarded appropriate If:

a) It was started as an empirical therapy according to IDSA guidelines.

b) It was switched to a narrow spectrum alternative antibiotic after culture and sensitivity data was obtained.

c) It was discontinued once the culture data were negative.

d) It was discontinued once the culture data showed a resistant organism.

e) The dosing, dose adjustments (if any) and indications were in accordance to a drug data base reference (Lexicomp).

If anyone of the described conditions was not met, the prescription was considered as inappropriate.

\section{Data collection and Analysis}

The medical record numbers of patients prescribed with Piperacillin/Tazobactam were retrieved from HIS, a computer based hospital wide information system. Patient data related to the demographics, diagnosis and type of Piperacillin/ Tazobactam therapy prescribed (empiric/definitive) was gathered. The relevant data was transferred to a SPSS version 16 which was then used to analyze the data by applying Pearson chi-square test.

\section{Results}

Table 1: Conditions for which Piperacillin/Tazobactam was prescribed empirically and the rationality of prescriptions.

\begin{tabular}{|c|c|c|c|c|}
\hline Interim Diagnosis & $\begin{array}{c}\text { Total number of } \\
\text { Prescriptions (Column) }\end{array}$ & Rational Prescriptions & Irrational Prescriptions & $\begin{array}{c}\text { Total number of } \\
\text { Prescriptions (Row) }\end{array}$ \\
\hline FN & $34(44.73 \%)$ & $29(85.29 \%)$ & $5(14.70 \%)$ & $34(100 \%)$ \\
\hline Sepsis & $19(26.02 \%)$ & $12(63.15 \%)$ & $7(36.84 \%)$ & $19(100 \%)$ \\
\hline UTI & $7(9.21 \%)$ & $5(71.42 \%)$ & $2(28.57 \%)$ & $7(100 \%)$ \\
\hline Pneumonia & $7(9.58 \%)$ & $2(28.57 \%)$ & $5(71.42 \%)$ & $7(100 \%)$ \\
\hline
\end{tabular}




\section{Global Journal of Pharmacy \& Pharmaceutical Sciences}

\begin{tabular}{|c|c|c|c|c|}
\hline WSSSTI & $3(4.10 \%)$ & $0(0.00 \%)$ & $3(100 \%)$ & $3(100 \%)$ \\
\hline Abdominal Infections & $6(8.21 \%)$ & $1(16.66 \%)$ & $5(83.33 \%)$ & $6(100 \%)$ \\
\hline Total & $76(100 \%)$ & $49(64.47 \%)$ & $27(35.52 \%)$ & $76(100 \%)$ \\
\hline
\end{tabular}

During the course of study, 76 empiric prescriptions were reviewed for 73 patients. The mean age of the patient was 36.34 years and $53.4 \%$ (39/73) were males. Table 1, is representing the indications for which Piperacillin/Tazobactam was used as empiric treatment for patients with suspected infections. The main indication, for which Piperacillin/Tazobactam was prescribed for, was FN (34/76; 44.73\%). The overall rates

of rational and irrational use of Piperacillin/Tazobactam for empirical therapy were $49 / 76$ (64.47\%) and 27/76(35.52\%) respectively, having P- value 0.001 . Most of the irrational prescriptions were in the cases of WSSSTI $3 / 3(100 \%)$, abdominal infections 5/6 (83.33\%), and pneumonia5/7 (71.42 \%) (Table1).

Table 2: Summary of Piperacillin/Tazobactam; Rational usage.

\begin{tabular}{|c|c|c|}
\hline Criteria & Rational & Irrational \\
\hline Started as an empiric therapy in accordance with IDSA guidelines & $49 / 76(64.47 \%)$ & $27 / 76(35.52 \%)$ \\
\hline $\begin{array}{l}\text { De-Escalation to an alternative after culture data showed sensitivity to narrow spectrum } \\
\text { antibiotic }\end{array}$ & $12 / 24(50 \%)$ & $12 / 24(50 \%)$ \\
\hline Discontinuation of therapy after clinical resolution and negative culture & $4 / 5(80 \%)$ & $1 / 5(20 \%)$ \\
\hline Discontinuation once the culture data showed Piperacillin/Tazobactam resistant organism & $7 / 8(87.5 \%)$ & $1 / 8(12.5 \%)$ \\
\hline \multirow{5}{*}{$\begin{array}{l}\text { Dosing, dose adjustments and indication in accordance to a drug data base reference } \\
\text { (Lexicomp). }\end{array}$} & $26 / 39(66.66 \%)$ & $13 / 39(33.33 \%)$ \\
\hline & Right Indications: & Wrong Indications: \\
\hline & $23 / 26(88.46 \%)$ & $11 / 13(84.61 \%)$ \\
\hline & $\begin{array}{c}\text { Dose adjustment done: } \\
\qquad 3 / 26(11.53 \%)\end{array}$ & $\begin{array}{c}\text { Wrong Frequency: } \\
2 / 13(15.38 \%)\end{array}$ \\
\hline & Total: $26 / 26(100 \%)$ & Total: $13 / 13(100 \%)$ \\
\hline
\end{tabular}

Table 2 describes the rational use of Piperacillin/Tazobactam keeping in view the criteria set to identify whether the usage was rational. Culture susceptibility and sensitivity work up was carried out for $45 / 76(59.2 \%)$ cases. Out of these 45 cases culture sensitivity test was returned positive for $40 / 45(88.9 \%)$ and negative for 5/45(11.11) cases. The most frequent organisms encountered were GNB including E.coli, Klebsiella.pneumoniae, Pseudomonas.aerugnosa, Acinetobacter species and related organisms. Culture sensitivity data revealed that organisms resistant to Piperacillin/Tazobactam were found in 8/40 cases (20\%) and sensitive to Piperacillin/Tazobactam as well as other alternative narrow spectrum antibiotics in $24 / 40$ cases (60\%).

However, it was inferred from the result that in $8 / 40(20 \%)$ cases, culture isolates sensitive not only to Piperacillin/ Tazobactam but also to other broad-spectrum antibiotics (meropenem, imipenem, cefepime).12/24 prescriptions (50\%) were de-escalated to a narrow spectrum antibiotic after culture sensitivity reports were received and reviewed. On the other Table 3: Pattern of distribution of empiric prescriptions of Piperacillin/Tazobactam in different units/wards.

\begin{tabular}{|c|c|c|c|c|}
\hline Unit & Total (prescriptions) & Rational prescriptions & Irrational prescriptions & Total (Rows) \\
\hline Surgical Oncology & $8(10.52 \%)$ & $6(75 \%)$ & $2(25 \%)$ & $8(100 \%)$ \\
\hline Pediatric Oncology & $26(34.21 \%)$ & $19(73.07 \%)$ & $7(26.92 \%)$ & $26(100 \%)$ \\
\hline Adult Oncology & $28(36.84 \%)$ & $18(64.28 \%)$ & $10(35.71 \%)$ & $28(100 \%)$ \\
\hline
\end{tabular}

hand in $12 / 24$ cases (50\%), organisms were sensitive to a narrow spectrum antibiotic, even then therapy was not changed, thus reflecting an irrational usage of the antibiotic. From 5/45 cases which were negative for cultures, in $4 / 5$ (80\%) prescriptions Piperacillin/Tazobactam was stopped and only $1 / 5$ prescription (20\%) was continued with Piperacillin/Tazobactam.

About 39/76 prescriptions (51.31\%) were evaluated on the basis of criteria of dose, dosing adjustments and right indications. Result depicted that $26 / 39$ orders $(66.66 \%)$ were found to meet the criteria for rational use whereas $13 / 39$ prescriptions $(33.33 \%)$ were irrational $(\mathrm{P}=0.000)$. Dosing and dose adjustments were done in a perfect way in all patients. Patients were distributed in different wards/units. The units in which most of the irrational prescriptions were ordered include palliative care unit (83.83\%) and ICU (37.5\%). Table 3 summarizes the distribution of patients in different units with respect to the rational use of Piperacillin/Tazobactam. 


\section{Global Journal of Pharmacy \& Pharmaceutical Sciences}

\begin{tabular}{|c|c|c|c|c|}
\hline Palliative care & $6(7.89 \%)$ & $1(16.66 \%)$ & $5(83.83 \%)$ & $6(100 \%)$ \\
\hline ICU & $8(10.52 \%)$ & $5(62.5 \%)$ & $3(37.5 \%)$ & $8(100 \%)$ \\
\hline Total & $76(100 \%)$ & $49(64.47 \%)$ & $27(35.52 \%)$ & $76(100 \%)$ \\
\hline
\end{tabular}

\section{Discussion}

Irrational utilization of Piperacillin/Tazobactam as compared to other broad-spectrum antibiotics, which increases the chances of emergence of resistant species [10], is a major contributing factor in the rise of resistance in gram-negative organisms as shown in a study. The increase in the number of empiric prescriptions and the up trending resistance, prompted us to conduct a study at our hospital. This study illustrate the statistically significant results $(\mathrm{P}=0.001)$ in case of rational and irrational prescription.

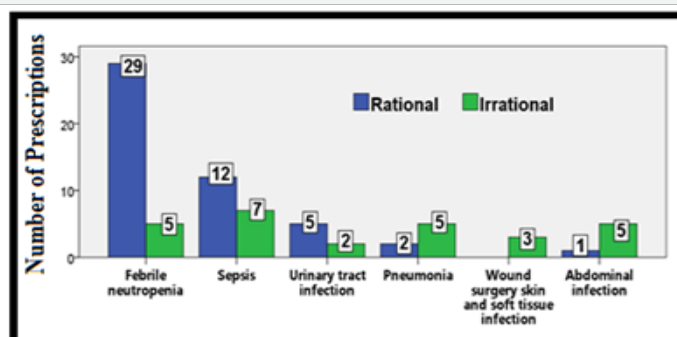

(A) Interim Diagnosis

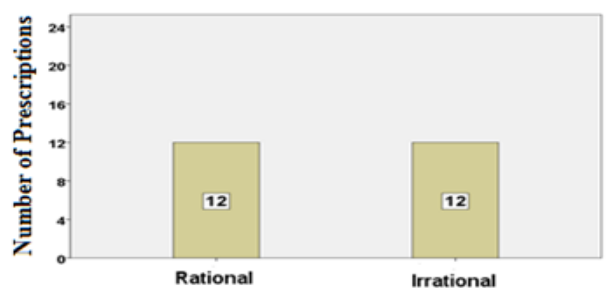

(C) Piperacillin/Tazobactam

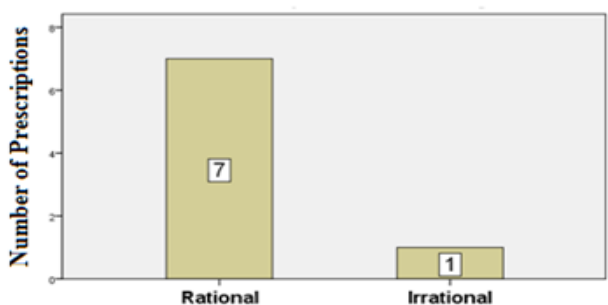

(E) Piperacillin/Tazobactam

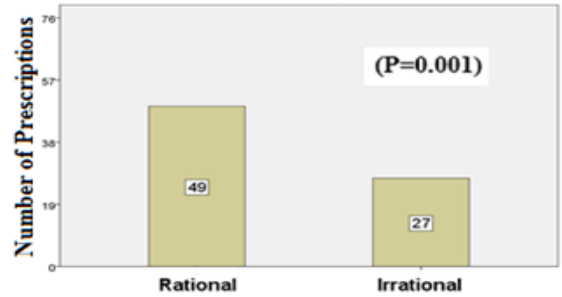

(B) Piperacillin/Tazobactam

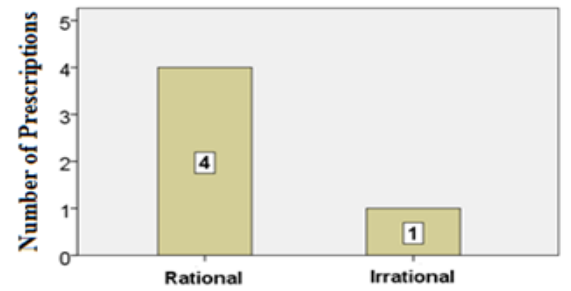

(D) Piperacillin/Tazobactam

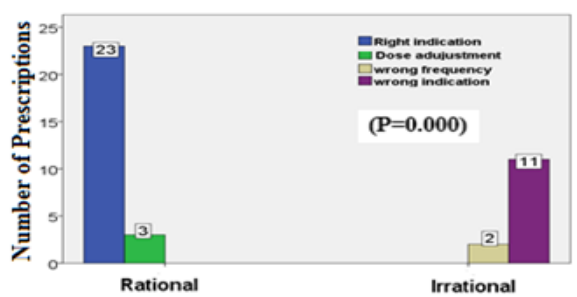

(F) Piperacillin/Tazobactam

Figure 1: Drug utilization review of Piperacillin/Tazobactam. Part (A), Piperacillin/Tazobactam started as empiric therapy in different diagnosis. Part (B), overall rational use of Piperacillin/ Tazobactam as empiric therapy in accordance with IDSA guidelines. Part (C), De-escalation to an alternative antibiotic after culture data showed sensitivity to narrow spectrum antibiotic. Part (D), Discontinuation of therapy after clinical resolution and negative cultures. Part (E), Discontinuation once culture data showed Piperacillin/Tazobactam resistant organism. Part (F), Dosing dose adjustment and indication in accordance to a drug database reference (Lexicomp).

Table1 for Piperacillin/Tazobactam as empiric therapy Figure 1 (Part: B).It is also revealed from the results Table 2, that according to the standard dosing, dose adjustments and indication, $26 / 39$ orders (66.66 \%) were found to meet the criteria for rational use whereas $13 / 39$ prescriptions $(33.33 \%)$ were irrational $(\mathrm{P}=0.000)$ Figure 1 (Part: $\mathrm{F})$.

As discussed earlier, number of bacterial cultures out of the positive ones for particular organism sensitive to Piperacillin/ Tazobactam as well as other alternative narrow spectrum antibiotics were $24 / 40(60 \%)$, out of these about $50 \%$ cases were possible to be switched to a narrow spectrum antibiotic but were continued with Piperacillin/Tazobactam. Moreover one subject grew a culture resistant to Piperacillin/Tazobactam, but the drug was continued.

Such findings may be attributed to lack of knowledge or negligence of the physician regarding culture results or antibiotic sensitivity profile [11], and it is clear and evident that modification of empiric therapy in accordance with the 
culture results will surely help in minimizing the cost, reducing the resistance, decreasing the trend of super-infections and improving the patient's quality of life [12].

The choice of any antibacterial agent depends not only on the identity of bacterial strain but also on the documented efficacy of drug in that particular infection, so most of the institutions are compelled to develop/follow a set of guideline which instruct about the empiric usage of the antibiotics. In our hospital, IDSA guidelines are followed. In addition to these guidelines, expert opinions from infectious diseases consultants, infectious diseases pharmacist and microbiologists are appreciated in about all clinical scenarios.

However a considerable number of irrational empirical prescriptions in the study are depicting either a lack of communication, education and implementation of these guidelines or uncertainty in the differential diagnosis, lack of confidence and experience of the attending physician and complex co-morbidities. Retrospective study being the major limitation, certain other limitations of this study include the lack of follow up for patients like those not switched to narrow spectrum antibiotic where it was possible or continued with Piperacillin/Tazobactam despite cultures were negative. The study also lacks comparison with any other published work due to lack of international data on Piperacillin/Tazobactam worldwide.

\section{Conclusion}

In appropriate Piperacillin-Tazobactam use has been observed at our hospital as evident by significant number of inappropriate empiric prescriptions regardless of the results of the microbial cultures and antibiogram pattern. Further prospective studies should be carried out to evaluate the cause of irrational prescribing.

\section{Recommendation}

We recommend:

a) To disseminate facility-specific criteria/guidelines via HIS, brochures and pamphlets.

b) To promote CME of the health care providers with a special emphasis on selection of appropriate empirical antibiotic therapy and modification once culture results are available.

c) To regularize constant surveillance for monitoring of the resistance patterns of important nosocomial bugs. d) To improve the utilization of Piperacillin/Tazobactam and patient outcome by applying method of preauthorization and prospective audit and feedback interventions by ASP team.

\section{References}

1. Laxminarayan R, Matsoso P, Pant S, Brower C, Røttingen JA, et al. (2016) Access to effective antimicrobials: a worldwide challenge. The Lancet 387 (10014): 168-175.

2. Liapikou A, Torres A (2016) Emerging drugs for nosocomial pneumonia. Expert Opinion on Emerging Drugs 21(3): 331-341.

3. Virizuela J, Carratalà J, Aguado J, Vicente D, Salavert M, et al. (2016) Management of infection and febrile neutropenia in patients with solid cancer. Clin Transl Oncol 18(6): 557-570.

4. Teng YN, Chang CS, Lee TE, Hung CC (2016) Cordycepin re-sensitizes multidrug resistance cancer cells to chemotherapeutic agents through modulating P-glycoprotein expression and ATPase function. Journal of Functional Foods 26: 681-690.

5. Van Hollebeke M, Chapuis C, Bernard S, Foroni L, Stahl J, et al. (2016) Compliance with carbapenem guidelines in a university hospital. Médecine et Maladies Infectieuses 46(2): 72-78.

6. Hughes WT, Armstrong D, Bodey GP, Bow EJ, Brown AE, et al. (2002) guidelines for the use of antimicrobial agents in neutropenic patients with cancer. Clinical Infectious Diseases 34(6): 730-751.

7. Shlaes DM, Gerding DN, John JF, Craig WA, Bornstein DL, et al. (1997) Society for Healthcare Epidemiology of America and Infectious Diseases Society of America Joint Committee on the Prevention of Antimicrobial Resistance: guidelines for the prevention of antimicrobial resistance in hospitals. Clin Infect Dis 25(3): 584-599.

8. Huh K, Chung DR, Park HJ, Kim M-J, Lee NY, et al. (2016) Impact of monitoring surgical prophylactic antibiotics and a computerized decision support system on antimicrobial use and antimicrobial resistance. Am J Infect Control 44(9): 145-152.

9. Khan FY, Elshafie SS, Almaslamani M, Abu-Khattab M, El Hiday AH, et al. (2010) Epidemiology of bacteraemia in Hamad general hospital, Qatar: a one year hospital-based study. Travel medicine and infectious disease 8(6): 377-387.

10. Dellit TH, Owens RC, Mc Gowan JE, Gerding DN, Weinstein RA, et al. (2007) Infectious Diseases Society of America and the Society for Healthcare Epidemiology of America guidelines for developing an institutional program to enhance antimicrobial stewardship. Clinical Infectious Diseases 44(2): 159-177.

11. Wagenlehner FM, Sobel JD, Newell P, Armstrong J, Huang X, et al. (2016) Ceftazidime-avibactam versus doripenem for the treatment of complicated urinary tract infections, including acute pyelonephritis: RECAPTURE, a phase 3 randomized trial program. Clin Infect Dis 63(6): 754-762.

12. Elhanan G, Sarhat M, Raz R (1997) Empiric antibiotic treatment and the misuse of culture results and antibiotic sensitivities in patients with community-acquired bacteraemia due to urinary tract infection. J Infect 35(3): 283-288. 
This work is licensed under Creative Commons Attribution 4.0 License

DOI: 10.19080/GJPPS.2017.03.555623
Your next submission with Juniper Publishers will reach you the below assets

- Quality Editorial service

- Swift Peer Review

- Reprints availability

- E-prints Service

- Manuscript Podcast for convenient understanding

- Global attainment for your research

- Manuscript accessibility in different formats

( Pdf, E-pub, Full Text, Audio)

- Unceasing customer service

Track the below URL for one-step submission https://juniperpublishers.com/online-submission.php 